\title{
Development of polyphenols-enriched maple sugars by freeze- and vacuum drum drying technologies
}

\author{
Bhatta, S. ${ }^{\mathrm{a}, \mathrm{b}^{*}}$; Stevanovic, T. ${ }^{\mathrm{a}, \mathrm{b}}$; Ratti, C. ${ }^{\mathrm{a}, \mathrm{c}}$ \\ ${ }^{a}$ Institute of Nutrition and Functional Foods (INAF), Laval University, QC G1V0A6 Canada. \\ b Renewable Materials Research Center (CRMR), Laval University, QC G1V0A6, Canada. \\ c Department of Soil and Agri-Food Engineering, Laval University, QC G1V0A6, Canada \\ *E-mail of the corresponding author: sagar.bhatta.1@ulaval.ca
}

\begin{abstract}
Hot water extract of sugar and red maple bark was added to maple syrup and dried by freeze (FD) and vacuum drum drying (VD) techniques. Addition of maple bark extracts to syrup helped to develop polyphenols-enriched maple sugar. X-ray diffraction revealed that sugar obtained from FD was amorphous in nature, while crystalline when dried by VD. Furthermore, the observation of maple sugar samples under scanning electron microscopy showed smooth and porous surface for FD sugar, while rough and grainy surface for VD sugar. Hausner ratio indicated that sugar produced by VD showed better flow characteristics than FD sugar.
\end{abstract}

Keywords: drying techniques; maple syrup; phenolics; microstructure 


\section{Introduction}

Drying of foods, solid (fruit and vegetables) or liquid (juices and syrups), increase their shelf life along with ease in handling and transportation. However, drying of foods cause the modifications of its physicochemical properties. In general, drying involving high temperatures effect thermo-labile compounds. Freeze drying (FD) is well-known technique for drying foods containing heat-sensitive and oxidative compounds such as polyphenols, because of the use of very low temperature and high vacuum. However, drying time and operation costs are major drawbacks of freeze drying [1]. Vacuum drum drying (VD) is another technique that operates at high temperature under vacuum, making it possible to dry food faster in comparison to freeze drying. Similar to FD, it may also preserve oxidative compounds due to the use of vacuum. Physical properties of powder such as moisture, flowability, morphology, etc depends on the type of drying method used [2, 3]. Therefore, drying techniques ought to be selected carefully, depending on the desired quality of the final product.

Canada accounts for approximately $71 \%$ of maple syrup production in the world (Statistics Canada, 2016). Maple syrup is a natural sweetener with high nutritional value based mainly on its sucrose, minerals, proteins and polyphenols [4]. Depending on the percentage of light transmission (at 560nm), maple syrups are classified into four grades: golden, amber, dark and very dark (Canadian Food Inspection Agency). A class of “very dark” maple syrup, which has light transmission less than $25 \%$, is considered as substandard. Therefore, drying of such substandard maple syrup to produce a maple sugar can add value to a natural sweetener containing minerals, polyphenols, etc. However, the polyphenols are present at a very low concentration in maple syrup. When dried, the concentration of polyphenols may further diminish. Therefore, the main objective of this work was to produce polyphenolenriched maple sugar by adding, red and sugar maple bark extracts to maple syrup, and subsequently drying the mixture.

For this study, freeze drying and vacuum drum drying technologies were explored. Thus obtained maple sugars were examined for total phenol content, moisture content, flow characteristics, crystallinity and morphology in order to study the effect of drying methods on developing the polyphenol-enriched sugars.

\section{Materials and Methods}

\subsection{Hot water bark extraction and addition of extracts to maple syrup}

Maple barks (sugar and red maple) were extracted using hot water following the method previously described by Geoffroy et al. [5]. Hot water extracts of maple barks were then added (at $0.01 \% \mathrm{w} / \mathrm{v}$ ) to maple syrup (very dark, $66^{\circ} \mathrm{Brix}$ ) and subsequently dried. Maple 
syrup without any extract was considered as control. Hereafter, dried maple syrup (without extract, control) will be denoted as MS, while maple syrup with sugar and red maple bark extract are identified as MS-SX and MS-RX, respectively.

\subsection{Drying experiments}

Freeze drying (FD) was done by using a laboratory freeze dryer (Freeze Mobile 24, Virtis Company Inc., Gardiner, NY). Sample was frozen at $-40{ }^{\circ} \mathrm{C}$ overnight and freeze-dried for 25h. The heating plate temperature was initially set at $-36^{\circ} \mathrm{C}$, vacuum level was below 30 mtorr and the condenser at $-90^{\circ} \mathrm{C}$.

For this study, a laboratory-scale vacuum drum dryer (Buflovak, Buffalo, USA) was used. Sample was fed between the nips of two rotating drums, where drum temperature increased up to $110^{\circ} \mathrm{C}$. The dried maple sugar powder were scraped off using in-built scrapers.

The obtained FD and VD samples were vaccum packed and stored in $4{ }^{\circ} \mathrm{C}$ for further analyses.

\subsection{Analyses of maple sugar powder}

\subsubsection{Total phenolic content}

Total phenolic content (TPC) in maple sugar powder was determined using the FolinCiocalteu method using microplate spectrophotometer (X Mark ${ }^{\mathrm{TM}}$, BIO-RAD) following the method used by Zhang et. al [6]. The results were expressed in milligram gallic acid equivalent per gram of oven dry sample (mg GAE/100g sample, db). TPC was measured in triplicates.

\subsubsection{Moisture content}

Moisture content of maple sugar powder was measured by vacuum-oven method at $70{ }^{\circ} \mathrm{C}$ and -22 in.Hg vacuum for $24 \mathrm{~h}$. Analyses were done in triplicates.

\subsubsection{Flow properties}

Hausner ratio (HR) was used to obtain the indication on flow characteristic of maple sugar powders. HR was calculated using the measured bulk and tapped density of the sample. Bulk density was measured by pouring the known mass of sample $(m)$ to a cylindrical glass tube and volume occupied $\left(V_{b}\right)$ was noted. For the tapped density, the glass tube was tapped from the vertical distance of $12 \pm 2 \mathrm{~cm}$ until no successive differences in volume $\left(V_{t}\right)$ was recorded. Bulk and tapped densities were calculated using the following formula; $m / V_{b}$ and $\mathrm{m} / V_{t}$, respectively. Measurements were done in triplicates. 


\subsubsection{X-ray powder diffraction}

The crystallinities of freeze and vacuum dried maple sugars were analyzed by powder Xray diffractometer (SIEMENS/Bruker, Germany). Samples were measured at a diffraction angle $(2 \theta)$ between 0 to $50^{\circ}$ under operational conditions of $40 \mathrm{kV}$ and $40 \mathrm{~mA}$ using Co $\left(\mathrm{K} \alpha_{1+2}\right)$ radiation.

\subsubsection{Microstructural analysis by environmental scanning electron microscopy (ESEM)}

Freeze and vacuum dried maple sugar samples were analyzed by environmental scanning electron microscopy (ESEM). The samples were scanned at $500 \mathrm{X}$ magnification at $20 \mathrm{kV}$.

\section{Results and Discussion}

\subsection{Effect of drying on total phenolics of maple sugar powder}

Regardless of the type of drying techniques used, TPC of sugars containing maple bark extracts showed higher values than the control. Sugar containing red maple bark extract presented higher TPC value than other sugar samples. This can be explained by the higher phenolic content of the red maple bark extract [7]. MS-RX obtained from VD had around 8\% higher TPC than the control (MS). In general, FD method is preferred to dry a food containing a thermo-labile compound such as phenolics. On contrary, we observed that VD maple sugars had relatively higher TPC than those of FD sugars. This could be due to high temperature in VD that could have caused the breaking of the phenolic-glycoside bonds. Resulting in more phenolics becoming readily available to form complex with Folin's reagent while determining TPC by Folin-Ciocalteu method. However, it has yet to be validated by identifying the phenolic profile in maple sugar powders using techniques of chromatography and mass spectrophotometry.

\subsection{Moisture content}

Moisture content of freeze-and vacuum dried maple sugars are presented in Fig. 1. The moisture content in VD maple sugar (0.6-0.7\%, on dry basis) was signigicantly lower than that of FD (4 to $5 \%$, dry basis). The drying temperature in VD was higher $\left(110{ }^{\circ} \mathrm{C}\right)$ than in FD. Therefore, it was obvious that VD produced sugars had lower moisture content than that of FD. Similar tendency was also observed for the production of mango powder by freeze and drum drying [8]. 


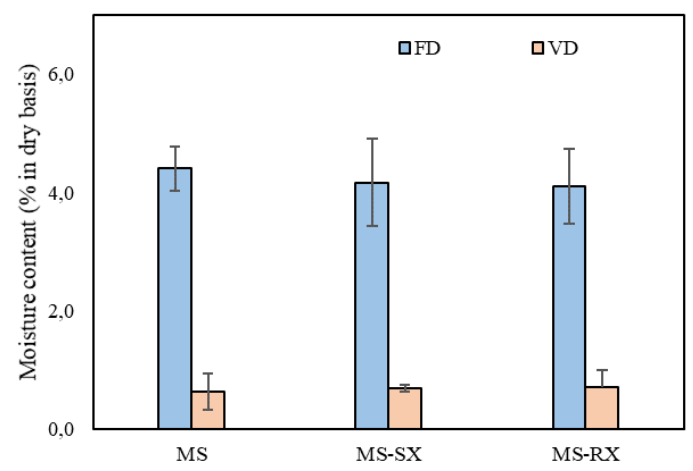

Fig. 1 Moisture content of freeze-and vacuum drum dried maple sugars. MS: maple sugar, MS-SX: maple sugar with sugar maple bark extract, $M S-R X$ : maple sugar with red maple bark extract, FD: freeze-drying, VD: vacuum drum drying.

\subsection{Flow characteristics of maple sugar}

The calculated Hausner ratio of FD and VD maple sugars are presented in Table 1. Lower the Hausner ratio, the better is the flow properties [3]. The Hausner ratio was signigicantly higher $(\mathrm{P}<0.05)$ for the FD maple sugars $(1.45$ to 1.48 , poor to very poor) than that for $\mathrm{VD}$ sugars (1.14 to 1.20 , good to fair flow). Therefore, VD maple sugars showed better flow characteristic than that of FD.

Table 1. Flow characteristics of freeze-and vacuum dried maple sugars.

\begin{tabular}{ccc}
\hline Drying techniques & Sample & Hausner ratio \\
\hline \multirow{2}{*}{ FD } & MS & $1.45 \pm 0.03^{\mathrm{a}}$ \\
& MS-SX & $1.48 \pm 0.07^{\mathrm{a}}$ \\
& MS-RX & $1.47 \pm 0.06^{\mathrm{a}}$ \\
\cline { 2 - 3 } & MS & $1.14 \pm 0.02^{\mathrm{b}}$ \\
VD & MS-SX & $1.20 \pm 0.04^{\mathrm{b}}$ \\
& MS-RX & $1.20 \pm 0.05^{\mathrm{b}}$
\end{tabular}

Values represent Mean \pm S.D. ${ }^{a, b}$ different superscript letters in the same column are significantly different $(P<0.05)$ by Tukey's test. 


\subsection{Effect of drying on crystallinity of maple sugar}

Fig. 2 depicts the profile of X-ray diffractogram of maple sugars produced by FD and VD techniques. Amorphous material shows large and disperse peaks due to disorderly arraged molecules in amorphous state. On the other hand, crystalline material shows sharp and defined peaks since the molecules are present in a highly ordered state. From Fig. 2, it can be observed that freeze dried maple sugar (FD_MS) is in amorphous state, whereas vacuum dried maple sugar (VD_MS), in crystalline state. However, the degree of crystallinity has yet to be identified. Crystalline state is important for the stability of food powders. Considering this results, VD sugars seemed to have an advantage of stability.

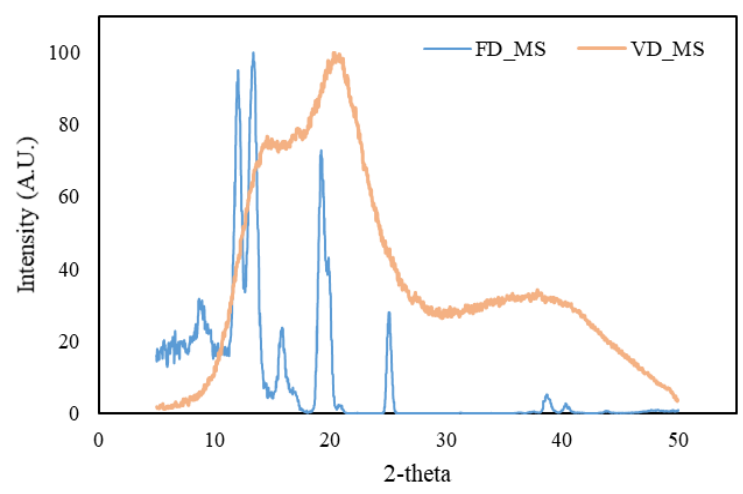

Fig. $2 X$-ray powder diffractogram of maple sugars produced by different drying methods. FD_MS, freeze dried maple sugar, VD_MS: vacuum dried maple sugar.

\subsection{Microstructural analysis}

Microstructural analysis of maple sugar obtained from FD and VD techniques are shown in Fig. 3. FD maple sugar showed irregular and porous structure (Fig. 3a). Freeze-dried food normally shows porous structure due to the fact that ice removal by sublimation during drying prevents shrinkage and volume reduction [1]. Vacuum-dried maple sugar resulted in rough and grainy surface (Fig. 3b). The difference in microstructure of FD and VD maple sugars can also be related to the the difference in their crystallinity (shown in Fig. 2). 


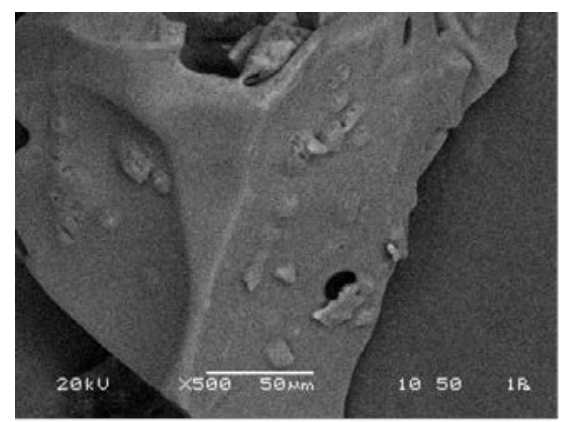

a

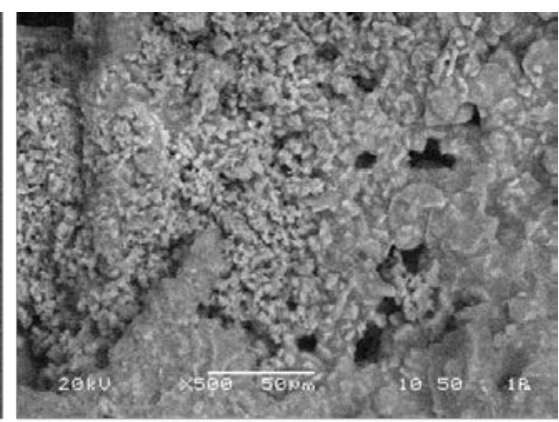

b

Fig. 3 Enviromental scanning electron micrograph (ESEM) of freeze-dried (a) and vacuum dried (b) maple sugar.

\section{Conclusions}

Considering the overall results, polyphenol-enriched maple sugar obtained either by FD or VD have potential applications in foods, particularly as sweetener for natural food products. For instance, FD maple sugar could be used in instant-drinks owing to its amorphous (powder) nature and VD sugar as functional food ingredient due to its high phenolic content as well as its better flow characteristics.

\section{References}

[1] Ratti, C. Hot air and freeze-drying of high-value foods: A review. Journal of Food Engineering 2001, 49 (4), 311-319.

[2] Jaya, S.; Das, H. A vacuum drying model for mango pulp. Drying Technology 2003, 21 (7), 1215-1234.

[3] Seerangurayar, T.; Manickavasagan A.; Al-ismaili, A. M.; Al-mulla, Y. A. Effect of carrier agents on flowability and microstructural properties of foam-mat freeze dried date powder. Journal of Food Engineering 2017, 215, 33-43.

[4] Ball, D. W. The Chemical Composition of Maple Syrup. Journal of Chemical Education 2007, 84 (10), 1647.

[5] Geoffroy, T. R.; Fortin, Y.; Stevanovic, T. Hot-water extraction optimization of sugar maple ( Acer saccharum marsh.) and red maple ( Acer rubrum l.) bark applying principal component analysis. Journal of Wood Chemistry \& Technology Feb. 2017, 1-12.

[6] Zhang, Q.; Zhang, J.; Shen, J.; Silva, A.; Dennis, D. A.; Barrow, C. J. A simple 96well microplate method for estimation of total polyphenol content in seaweeds. 
Journal of Applied Phycology 2006, 18 (3-5), 445-450.

[7] Bhatta, S.; Ratti, C.; Poubelle, P. E.; Stevanovic, T. Nutrients, Antioxidant Capacity and Safety of Hot Water Extract from Sugar Maple ( Acer saccharum M .) and Red Maple ( Acer rubrum L .) Bark. Plant Foods for Human Nutrition 2018, 73 (1), 2533.

[8] Caparino, O. A.; Tang, J.; Nindo, C. I.; Sablani, S. S.; Powers, J. R.; Fellman, J. K. Effect of drying methods on the physical properties and microstructures of mango ( Philippine ' Carabao ’ var .) powder. Journal of Food Engineering 2012, 111 (1), 135-148. 